\title{
Patient periprocedural stress in cardiovascular medicine: friend or foe?
}

\author{
Paweł Iwaszczuk ${ }^{1}$, Władysław Łosiak², Wojciech Szczeklik², Piotr Musiałek ${ }^{1}$ \\ ${ }^{1}$ Department of Cardiac and Vascular Diseases, John Paul II Hospital in Krakow, Institute of Cardiology, Jagiellonian University \\ Medical College, Krakow, Poland \\ ${ }^{2}$ Department of Philosophy, Institute of Psychology, Jagiellonian University, Krakow, Poland \\ ${ }^{3}$ Center for Intensive Care and Perioperative Medicine, Jagiellonian University Medical College, Krakow, Poland
}

Adv Interv Cardiol 2021; 17, 3 (65): 259-271

DOI: https://doi.org/10.5114/aic.2021.109176

\begin{abstract}
A bstract
Stress, a disruption of homeostasis, is an unavoidable part of everyday life. In medical procedures, stress profoundly affects both operators and patients. Although the stress reaction has evolved to aid survival of physical trauma, it may also be harmful, by aggravating the baseline medical condition and/or creating new stress-related medical problems. Stress responses comprise several protective mechanisms that are particularly relevant in the clinical setting (e.g., a procoagulatory state and blood loss counteraction, preservation of blood perfusion pressure, prevention of hypoglycemia, enhanced immune response). Beneficial psychological effects prevent recurrence of traumatic memories, and promote patient compliance and positive lifestyle changes. In contrast, overt acute stress responses may lead to severe pathological conditions such as cytokine storm, post-traumatic stress disorder, takotsubo syndrome, deep venous thrombosis and pulmonary embolism, myocardial infarction, life-threatening arrhythmias and sudden cardiac death. There is also evidence that stress exposure may promote atherosclerosis and reduce long-term benefits from the intervention (increase in major adverse clinical events, in-stent restenosis, etc.). Insights into the role of stress on the operator's performance have recently led to the introduction of counteractive measures such as simulation training. Conversely, very little is known about the effect of the patient's periprocedural stress on the outcomes of cardiovascular procedures. Recent data show that the patient periprocedural stress affects the well-being of whole families. This review, focused on topics particularly relevant to cardiovascular interventions, provides a mechanistic insight into beneficial and harmful effects of periprocedural patient stress, including the array of available stress-relieving measures.
\end{abstract}

Key words: management, stress, mechanisms, effects, cardiovascular interventions.

\section{Introduction}

Adaptive neurohormonal changes in response to stressful situations have evolved as protective mechanisms promoting survival in severe circumstances. These pathways are also activated (to a lesser extent) to aid an organism during everyday simple tasks [1]. On the other hand, intense acute emotional stress can cause severe medical conditions such as post-traumatic stress disorder (PTSD), takotsubo syndrome (TTS), deep venous thrombosis and pulmonary embolism (DVT/PE), and trigger myocardial infarction (MI), life-threatening arrhythmias and sudden cardiac death (SCD) [1-3]. Recent evidence indicates that the psycho-neurogenic component of the stress response is at least partially responsible for cardiac contractility impairment in one fourth of patients newly diagnosed with acute coronary syndrome (ACS) [4]. The effects of long-term psychological stress have been proven detrimental for human health. Apart from psychiatric sequelae such as depression, burnout, chronic fatigue or substance use (including smoking), chronic stress causes immunologic perturbations predisposing to more frequent infections and cancer [5-7], increases hunger and food intake ("stress eating") leading to obesity [8], insulin resistance, diabetes and possibly hypertension (the metabolic syndrome) [9], and thus, ultimately promotes atherosclerosis [10]. The vasospasm and procoagulatory state evoked by acute stress, which include increase in hematocrit, fibrinogen, von Willebrand factor and several other plasmatic coagulation factors [2, 11], have (assumingly - as per the evolutionary approach)

\section{Corresponding author:}

Paweł Iwaszczuk BSc, MD, Department of Cardiac and Vascular Diseases, John Paul II Hospital, Institute of Cardiology, Jagiellonian University Medical College, Krakow, Poland, phone: +48 1261422 87, e-mail: p.iwaszczuk@gmail.com

Received: 24.05.2021, accepted: 17.06.2021. 
beneficial effects for victims of severe physical trauma, as they help maintain hemostasis [12]. However, the same mechanisms (intertwined with complement and other inflammatory systems) may cause arterial thrombosis upon atherosclerotic plaques [11], DVT/PE as shown by epidemiologic data from natural disasters [2], or even lead to a disseminated intravascular coagulation (DIC) syndrome in response to a strong inflammatory stressor as in severe sepsis or polytrauma [12].

Surgical and endovascular procedures are associated with various degree of physical insult, as well as emotional stress inflicted on the patient, which is linked to anticipation of the procedure and possible complications, as well as intra- and post-procedural stressful stimuli in conscious, non-sedated patients. The net effect of this periprocedural stress has not been established. However, numerous medications (including those frequently used) affect the perioperative stress responses.

The purpose of this review is to summarize positive and negative aspects of stress reaction in a patient undergoing invasive procedures, to discuss current means of controlling these responses, to highlight the importance of stress-related cardiomyopathy syndromes that are of particular interest to interventional cardiologists, and finally, to provide clinicians with an update on advances in understanding the stress response derived from neuroscientific research and to point out directions for future clinical studies. A Medline search for "stress" provides ca. 50000 results annually in recent years, and thus, this review will cover mainly the qualitative aspects.

\section{Definition and varieties of stress}

Below, we provide contextual boundaries and the most commonly used nomenclature. Etymologically, the word stress is derived from Latin districtus (torn, hindered) and Old French estrece (narrowness, oppression), through thirteenth century Middle English distresse - later shortened to stresse (hardship, adversity, force, pressure) $[2,13]$. Despite its previous occasional use in colloquial speech to describe a difficult situation or in physics (mechanics) to describe forces that lead to strain, stress was adapted to psychobiology and popularized by the endocrinologist Hans Selye in the mid-twentieth century (although he initially preferred to name it general adaptation syndrome - GAS) [10]. He classified stress into two major categories: eustress, associated with positive emotions, and distress, associated with negative emotions, stating that the former has less detrimental consequences than the latter $[6,10]$. Later, Lovallo identified different patterns of activation in effector organs between the two, clearly reinforcing that positive emotions do not preclude stress, but also, that the reaction to challenge is not as unspecific as previously thought [10]. Still, some researchers limit their consideration of stress only to negative phenomena, distinguishing it from mobilization in response to positively challenging experiences
[10]. Dhabhar recommend a differentiation between the short-term (lasting minutes to hours) "good" stress and prolonged "bad" stress [6]. This distinction may, however, also be too simplistic, since short-term stress is the primary cause of stress cardiomyopathy (TTS) or PTSD. The most commonly cited two shades of stress cardiomyopathy in mainstream media include the broken heart syndrome (TTS in response to negative emotions) and the happy heart syndrome (TTS following strong positive emotions), both proven to be potentially deadly [14]. Finally the term stress is often interchangeably used to describe three associated but distinct phenomena - a demanding situation requiring adaptation (stress), stressful stimuli (stressors) and responses to them (stress reaction) [2], which is avoided in this paper.

The most common understanding embraces psychological (emotional) stress that originates from the brain limbic structures, where the vast sensory input is processed, perceived and categorized as harmful or not [1, 15]. The negative emotional and associated physiological response to truly or potentially noxious external stimuli is called fear; however, most researchers simplify their terminology to anxiety only, which comprises an exaggerated fear response, as well as reactions induced by factors that are not harmful by themselves but associated with stressful experiences in memory traces or originating from intrinsic brain activity (i.e., thoughts). Somatic (physical) stress is associated with physicochemical tissue injury, often called systemic when considered on a larger scale - affecting whole organs or the whole body, and is usually accompanied by psychological stress, because either pain or just the notion of damaged skin is sufficient to trigger an emotional stress response [15, 16]. However, many physical stressors act subconsciously (e.g., electrolytic disturbances), as they are detected by receptors that do not project to higher cortical regions, and seem to trigger the response via neural pathways distinct from those initiated by psychological stressors [15]. Additionally, some investigators limit their scope of stress consideration to specific circumstances such as social stress (a subcategory of psychological stress), exertional stress (associated with physical exercise) and oxidative or excitotoxic stress, which refer to physicochemical changes at the cellular and tissue level (subcategories of somatic stress) [2]. However, even a single cell remains in constant dynamic intrinsic and extrinsic equilibrium (homeostasis), and therefore stress can be broadly defined as the "actual or anticipated disruption of homeostasis or well-being" [15].

\section{Homeostasis, allostasis and allostatic load - overload}

In response to a stressor, an organism makes efforts to restore homeostasis or well-being, or to prevent their loss in advance [15]. A state of this new dynamic equilibrium is called allostasis, which means "achieving stabil- 
ity through change" [1], and is essentially an equivalent term for dynamic balance under the influence of a stressor. However, attaining allostasis is associated with neurohormonal storm and metabolic costs which take their toll upon the organism - the allostatic load [1, 17]. As shown schematically in Figure $1 \mathrm{~A}$, an ideally adequate stress response is an abstract construct, especially regarding psychological stress, since in reality the effectors of the stress reaction are usually activated in abundancy and stronger than necessary to achieve allostasis, have different degree of inertness, and are activated in anticipation, all of which constitute the allostatic load. When an indi- vidual suffers from "repeated hits" of different stressors, to which one is unable to adapt, these penalties accumulate (even if the stress response is fully physiologic) and generate the allostatic overload [1]. Other causes of allostatic overload shown in Figure $1 \mathrm{~B}$ include: lack of adaptation to a mild repeated stressor, a prolonged response without physiologic return to homeostasis after the stressor's withdrawal, a marked overactivation of stress effectors or profound underactivation in response to a particularly harmful stressor [1]. The result of allostatic overload depends greatly upon which component of the allostatic network is affected and how. For instance,

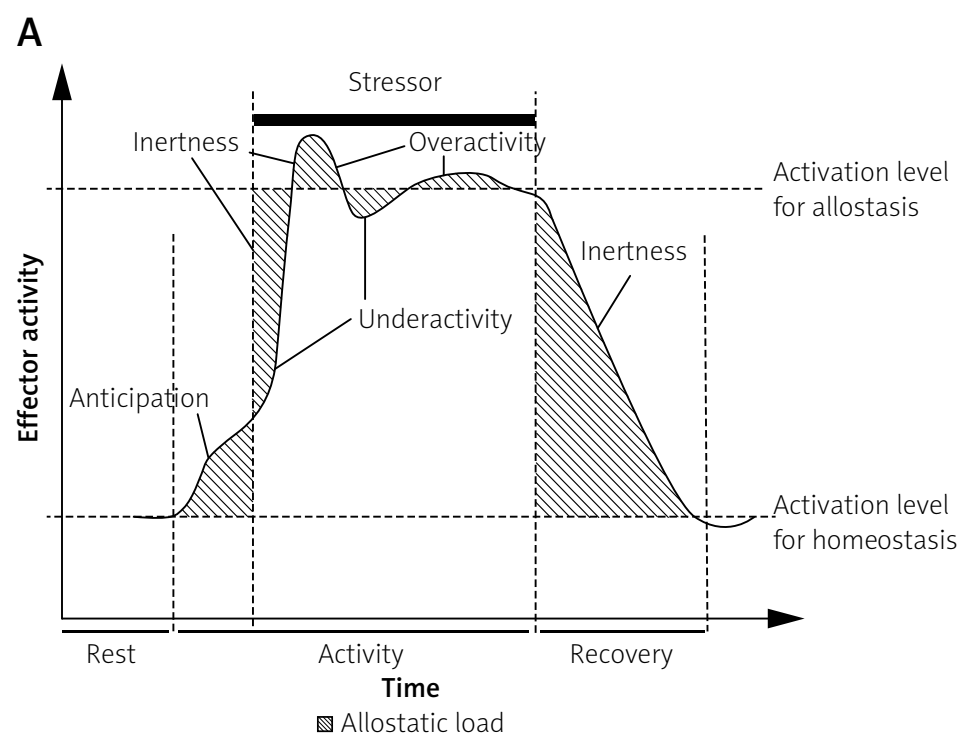

B
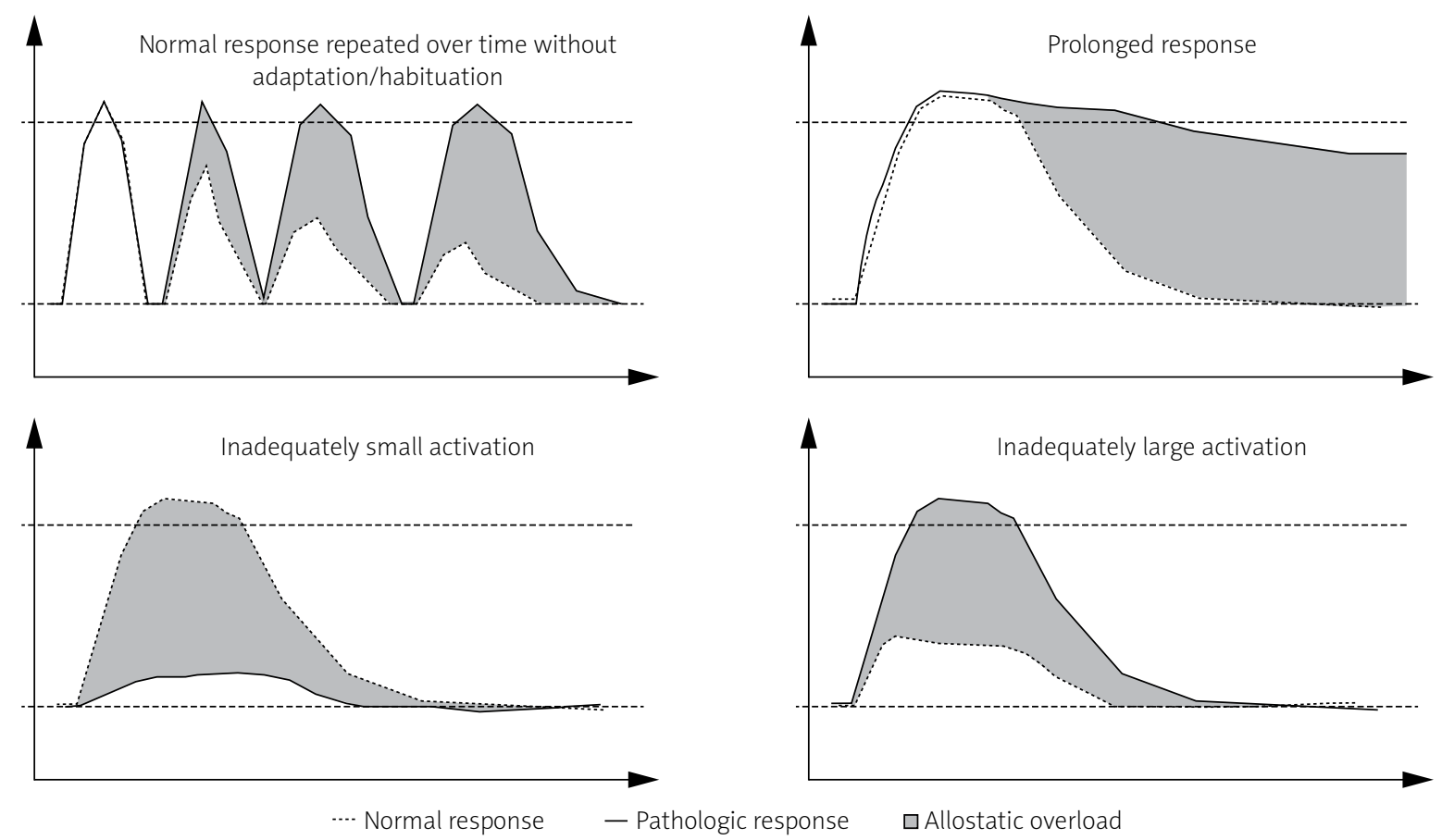

Figure 1. Schematic representation of physiologic (A) and pathologic (B) stress responses, explaining the origins of allostatic load and overload. Modified after [1, 52] 
overactivity of the HPA axis in the perioperative period is associated with prolonged wound healing and recovery, whereas in post-traumatic stress disorder (PTSD) it has been implied that marked underactivation of the HPA axis in response to powerful stressor is associated with inadequate suppression of memory trace formation in the hippocampus, leading to the associated flashbacks of the traumatic event [1]. The same HPA axis becomes overactive in various forms of chronic stress, contributing to its detrimental effects on health $[6-10,18,19]$. There is evidence suggesting presence of structural changes in the brain resulting from neuroplasticity in chronic stress $[1,15]$, leading to generally more pathological responses to stressors in chronically stressed individuals.

\section{Gross physiology of stress response:} the allostatic network

The stress response is a complex sequence of neuroendocrine changes in numerous interacting systems collectively known as the allostatic network, which embraces the two major effectors: hypothalamo-pituitary-adrenal (HPA) and sympatho-adreno-medullary (SAM) axes [1, 15]. Other important players in stress response include proand anti-inflammatory cytokines, sex hormones, and the parasympathetic division of the autonomic nervous system (PNS) which is in constant reciprocal balance with SAM $[1,15]$. This neurohormonal allostatic network exerts its effects on (and receives feedback from) the cardiovascular and immune systems, metabolic reactions and the brain, which is the central regulator of the stress response [1]. Glucocorticoids have complex non-genomic and genomic actions through higher-affinity mineralocorticoid receptors present only in selected tissues (such as brain, heart and kidneys), that are activated already under basal conditions, and through lower-affinity glucocorticoid receptors, which are present in virtually all nucleated cells, but become activated only during the morning peak cortisol release and under stress [1, 15]. The net result of HPA axis activation in a particular cell or organ varies greatly depending on the expression of these receptors, but also upon concentration of the agonist, which is further modulated by the bioavailability of cortisol (most is bound to corticoid binding globulin, whereas only the free fraction is biologically active) and time of glucocorticoid activity, all in all, creating a complex biphasic pattern of response, which can sometimes even become pro-inflammatory [1, 20]. Endogenous cortisol is conventionally believed to deliver at least four types of actions: permissive (basal cortisol levels augment the first wave of the stress response), stimulatory (stress cortisol levels enhance the magnitude of the first wave of the stress response), suppressive (stress cortisol levels inhibit through a feedback loop the first wave of the stress response to prevent an overshoot) and preparative (stress cortisol levels facilitate the physiologic re- sponse to subsequent/repeated stressors) [21]. The main neurotransmitters of the SAM axis in the effector organs comprise adenosine-triphosphate (ATP) activating immediate-acting ion channels, noradrenalin (NA) employing fast-acting G-coupled adrenergic receptors and slow-acting neuromodulators, among which neuropeptide Y (NPY) seems to be the most prominent [22-24]. The endocrine portion of the SAM axis - the adrenal medulla - releases adrenalin (A) and to a lesser extent other catecholamines into the blood stream $[15,22]$. The contradictory (but to some extent also complementary) PNS utilizes immediate-acting ATP to a lesser extent, exerting its actions mainly through fast-acting acetylcholine (ACh), intermediate-acting nitrous oxide (NO) and slow-acting modulators, most prominently vasoactive intestinal peptide (VIP) $[22,24]$. The net effect is dependent on mutual balance between SAM and PNS activity, as well as on the distribution of the receptors in target tissue, for instance the gradient of $\beta 1$ and $\beta 2$ adrenergic receptors in the heart [25]. The co-transmitters in SAM and PNS seem to play an important role in the disease pathophysiology; for instance, increased ATP signaling has been linked with hypertension, vascular remodeling in atherosclerosis and neointimal hyperproliferation following angioplasty, and heart failure, not to mention the potent effect of its immediate derivative (ADP) on platelet aggregation and activation [24]. Vagal afferents seem to detect inflammatory cytokines and mediate the information about local inflammation to the central nervous system, while the vagal efferent output seems to modulate splenic and hepatic immunologic responses (the inflammatory reflex) [26]. Overall, acute stress enhances innate and adaptive immunity, while chronic stress hinders immunity and contributes to the pathogenesis of numerous diseases [6, 10]. The complex physiology, psychology, as well as neuroanatomical basis and regulation of the stress response are largely beyond the scope of this review; however, a few issues will be pointed out further in the text. In Figure 2 we show a fairly detailed schematic diagram of the main neurophysiologic pathways of the stress response for reader's reference, and to illustrate the complexity, which extends far beyond the classically considered, anxiety-driven, HPA and SAM axes. In Table I we summarize the most common tools used to quantify stress.

\section{Stress of the operator}

The periprocedural medical setting is stressful to both the performer and the subject. The operator's performance is affected by stress in many ways. Mild to moderate stress may serve as positive mobilization and help focus on the task at hand. There is substantial evidence that excessive stress impairs surgical performance in both technical and non-technical skills (such as communication and decision making), and that this effect is more pronounced in less experienced surgeons [27]. 


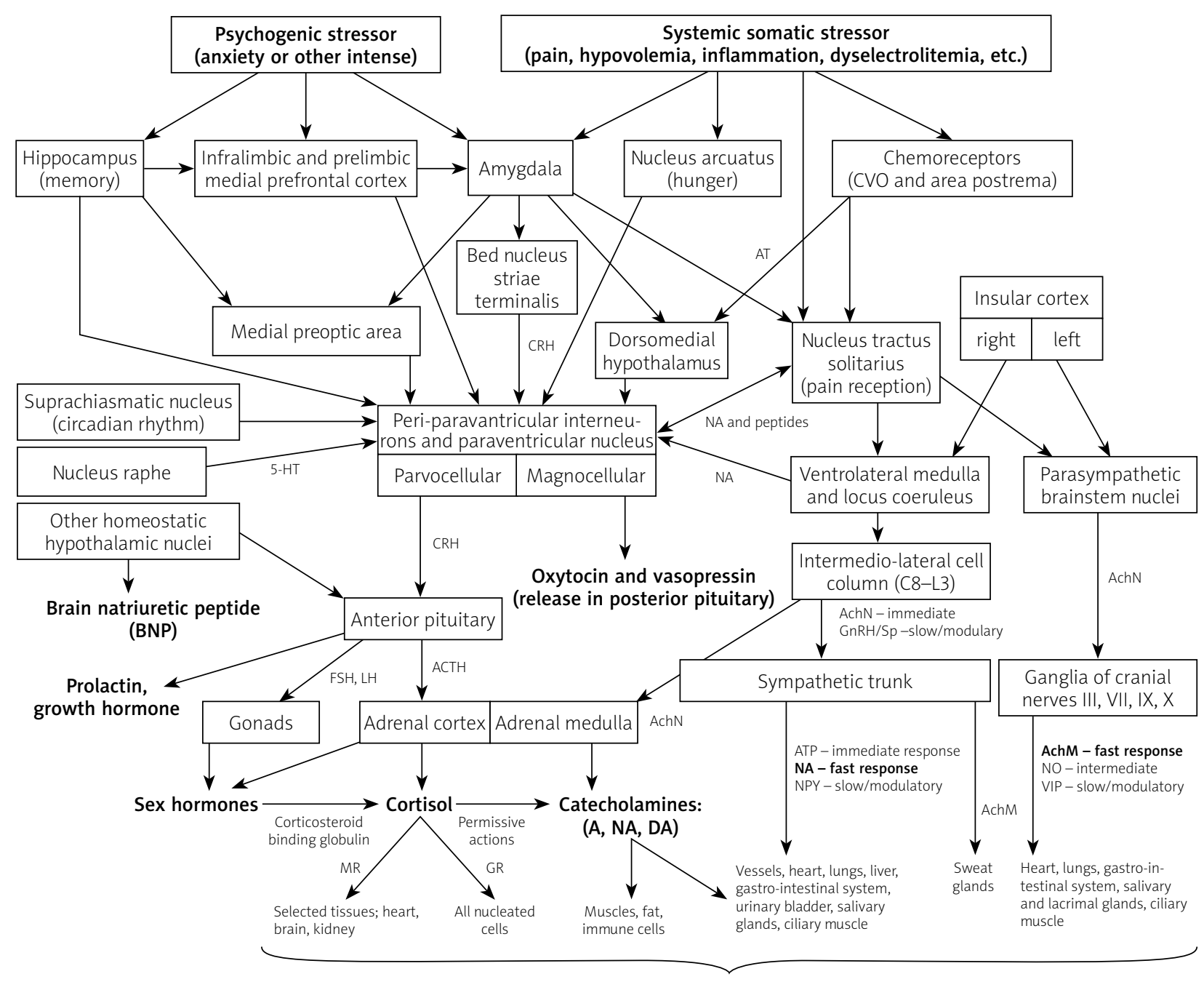

METABOLISM, CARDIOVASCULAR AND IMMUNE SYSTEMS

Figure 2. The allostatic network and its major regulatory pathways. The stress response is always initiated in the brain, but different stressors elicit distinct pathways of activation, which impacts the resulting net balance of the effectors. The complex of the paraventricular nucleus of the hypothalamus (PVN) is undoubtedly the central integrating point of all of the inputs, which initiates both HPA and SAM axes; however, it is the bed nucleus striae terminalis (BST) and dorsomedial hypothalamus (DMH) that link the major inputs with PVN, and therefore serve as a central management unit of the stress response. The fastest action is mediated via neural pathways of the autonomic nervous system (which seems to be coordinated by the insular cortices [25]) and, within (milli)seconds, reaches effector organs facilitating the fight-or-flight response. However, the subsequent intermediate and slow responses (due to the released co-transmitters) also seem very important for the end effect of activation. The most important transmitters in each branch are indicated in bold. The SAM axis, after the first hit from its nerve endings and reciprocal PNS withdrawal, triggers release and synthesis of new catecholamines in the adrenal medulla, which takes minutes. Even more inertly, the HPA axis takes tenths of minutes to fully enable its action, which can be generally perceived as protective and counteracting the SAM axis (despite many synergistic actions) and in physiologic conditions - in response to a short acting stressor - ultimately leads to waning of catecholamine and inflammatory allostatic load, and terminates the stress response via a negative feedback loop through the hippocampus. Other circulating hormones released from the hypothalamus, pituitary and gonads modulate the whole response in a complex manner

5-HT - 5-hydroxytryptamine (serotonin), A - adrenaline, AT - angiotensin II, NA - noradrenaline, DA - dopamine, AchM and AchN - acetylcholine acting via muscarinic and nicotinic receptors respectively, $A C T H$ - adrenocorticotropic hormone, $C R H$-corticotrophin releasing hormone, GR-glucocorticoid receptor, $M R$ - mineralocorticoid receptor, NPY - neuropeptide Y, NO - nitric oxide, FSH - follicle-stimulating hormone, GnRH - gonadotropin releasing hormone, LH - luteinizing hormone, $S P$-substance P, CVO - circumventricular organs (organum vasculosum of the lamina terminalis and subfornical organ). Drawn based on references: [1, 15, 22, 25, 52] 
Table I. Most common indicators used in stress research

Cortisol level:

- Salivary (free cortisol, good resolution, non-invasive)

- Blood (free and total cortisol, invasive)

- Urine (corrected for creatinine concentration or 24-hour collec tion; lower temporal resolution but generally more robust measurement, affected by renal function)

Catecholamine level:

- Blood (unstable, must be measured minutes after collection, transported on ice)

- Urine metabolites (easy measurement, same issues as with urinary cortisol)

Other stress-related hormones:

- BNP, angiotensin II, sex hormones, adrenal androgens, prolactin, vasopressin, oxytocin, growth hormone, glucagon, hypothalamic liberins, and many others (many confounding factors, modulators rather than main effectors of stress response)

Immunologic function:

- Cytokine measurements (difficult to interpret, due to the complex relationships)

- Epidemiologic data (indirect; e.g., wound healing, infection rate, cancer metastases, autoimmune disorders, etc.)

Blood pressure monitoring:

- Unreliable in hypertensive patients

Heart rate variability:

- Sympathetic and parasympathetic tone and reactivity (indirect measurement, many confounding factors, inapplicable in arrhythmias)

Other autonomic markers:

- Galvanic skin response (very good temporal resolution, no absolute values, just relative measurements)

- Respiratory rate and depth (prone to confounding factors, e.g., dyspnea, speaking)

Muscle tension:

- (Surface) electromyography of the so-called stress muscles (e.g., masseter, trapezius) may indicate stress-related tension (prone to movement confounders)

Psychological questionnaires:

- Anxiety (most common)

- Negative mood measured with adjective checklist

- Depression (indirect)

- Life events (valuable in identifying confounders, but time consuming)

- Coping strategies and personality traits (modulatory to stress responses)

- Other

Functional neuroimaging (fMRI, PET):

- Activation of stress-related neural pathways (difficult to implement in clinical setting, time consuming and expensive)

There is little research focusing particularly on stress affecting an interventional cardiologist during percutaneous procedures; however, laparoscopic surgical procedures have been shown to induce even greater stress levels than open surgery [27], and therefore it is reasonable to assume that the level of stress experienced by the operator during $\mathrm{PCl}$ is no less than in any other invasive medical procedure (or even higher), but further research is needed here.

The surgeon's stress has been recognized, studied and characterized to a fair extent. Furthermore, several countermeasure lines (such as simulator training) have been developed and are being implemented [27, 28]. In contrast, the patient's stress has been largely neglected in the consideration of outcomes. With the exception of anxiolytic premedication regimens, applied either routinely to all patients before a specified procedure or only to selected patients who explicitly show emotional distress (usually at the discretion of the anesthesiologist qualifying the patient for anesthesia or the operator himself, depending on the type of procedure and local practice), the stress of the patient is usually disregarded. Apart from scarceness of reliable tools to effectively and easily quantify the magnitude of stress suffered by the patient, as well as limited therapeutic options, probably the most important factor hindering any focus on the patient's stress is the obscure answer to the core question whether stress of the patient is ultimately more detrimental or beneficial to the outcome.

\section{Benefits of patient stress}

Several survival-promoting aspects of stress responses may play an important role in the outcomes of cardiovascular procedures.

\section{Lessons from adrenal insufficiency}

Adrenal insufficiency, a lethal condition that, even nowadays with treatment, may develop into adrenal crisis with a mortality rate of about 8/100 patient-years [29], illustrates the significance of cortisol-mediated responses to stress. Acute lack of glucocorticoids, when they are needed to balance the burden of other allostatic effectors, leads to death, most likely due to the lack of suppressive actions on inflammatory cytokines, in particular on secretion and sensitivity to tumor necrosis factor $\alpha$ (TNF- $\alpha$ ) with its ability to induce glucocorticoid resistance [29]. However, chronic cortisol depletion may also diminish the permissive glucocorticoid actions, so that the adrenal crisis manifests clinically in a nonspecific manner as a major deterioration in general health, exhaustion and depression followed by impaired cognition and somnolence, anorexia, nausea and vomiting (sometimes associated with abdominal pain mimicking early peritonitis) and profound hypovolemic hypotension, as well as other symptoms associated with the precipitating condition (especially diarrhea and fever, physical or emotional stressor in anamnesis) [29]. Blood test results usually show hypoglycemia in a non-diabetic, whereas other classically associated laboratory disturbances (hyponatremia, hyperkaliemia, mild normocytic anemia, lymphocytosis and eosinophilia) as well as hyperpigmentation and weight loss usually occur only in previously undiagnosed and chronically developing adrenal insufficiency, while a patient in an acute syndrome or with an established diagnosis and on treatment may enter an adrenal crisis within one to a few hours after encounter- 
ing a stressor [29]. Cardiologic evaluation in such cases, apart from hypovolemic shock resistant to infusion of crystalloids and pressor amines, may reveal electrocardiographic (ECG) and echocardiographic abnormalities suggesting acute heart failure [29].

\section{Protective aspects of stress}

The mechanisms through which stress may be beneficial in a periprocedural setting are summarized in Table II.

Circadian rhythm, an essential feature of cardiovascular parameters such as blood pressure or heart rate, plays a core role in regulation of stress responses [1]. The evolutionary concept of the stress response focuses on its crucial role in surviving injuries, especially those associated with marked hemorrhages [2, 12]. The stress response also serves as a potent immunomodulator numerous studies have shown that short-term-stressinduced immunomodulation can potentiate a positive response to vaccination, and some argued that it could have beneficial effects for immune responses towards cancer cells, as well as in surgery in general [6]. On the other hand, much research has demonstrated a relationship of the stress response during oncologic surgery (especially actions mediated via catecholamines and prostaglandins) with cancer metastasis formation and relapses of the disease [5]. These findings, however, are only seemingly contradictory, and a broader understanding of the impact of many intricacies in the perioperative stress response on the immune system is needed to potentially modulate these outcomes with a timely intervention [6]. Similarly, the adequate and timely HPA axis activation helps to facilitate stress-related learning, but also serves as a protective mechanism against PTSD, since its action in the precise time-frame in the hippocampus blocks consolidation of traumatic memories, and thus prevents flashbacks $[1,30]$. These powerful effects on memory and learning also underlie the potential to induce life changing behavior after $\mathrm{MI}$ or a major surgical procedure, or any other stressful medical encounter followed by recommendations on lifestyle [31]. Furthermore, acute stress has also been shown to facilitate prosocial behaviors in humans [23], which may also contribute to better compliance.

\section{Detriments of patient stress}

Table III summarizes fundamental detrimental effects of patient stress.

\section{Stress as ACS trigger and chronic myocardial ischemia exacerbator}

Emotional stress has been indicated as a causative factor for Ml since its first description in the medical literature, and even earlier in folk wisdom in expressions such as "broken heart" or "scared to death" [11, 32]. Higher prevalence of $\mathrm{MI}$ in the morning and on Mondays
Table II. Benefits of periprocedural patient stress (stress as a friend)

Sympatho-adreno-medullary (SAM) axis activation:

- Provides dynamic maintenance of cardiovascular stability:

- Increases heart rate and contractility, and hence, cardiac output

- Increases peripheral vascular resistance through vasoconstriction

- Increases baroreceptor sensitivity which facilitates cardiac rehabilitation

- A primary mechanism to sustain blood pressure and perfusion of organs

- Enhances immune function, especially innate immunity mechanisms

- Releases nutrients from storage

Hypothalamo-pituitary-adrenal (HPA) axis activation:

- Prevents immunologic overactivation and cytokine storm

- Induces protective state in cells through glucocorticoid receptors

- Releases nutrients from storage

- Suppresses traumatic memories (prevents PTSD)

- Potentially prevents development of pathologic adaptive immunity to own antigens present in cells and tissues, and thus from autoimmunization

- Terminates stress response through feedback loop

Procoagulatory state:

- Facilitates hemostasis

- Reduces blood loss

Stressful experience:

- Can help to elicit life-style changes

Table III. Detrimental effects of periprocedural patient stress (stress as a foe)

Hyperthrombotic state:

- Hyper-enhanced blood clot formation is associated with the development of thrombosis (DVT/PE) as well as STEMI, and may also be associated with other thromboembolic complications (e.g., ischemic stroke, acute limb ischemia)

- In some conditions can precipitate disseminated intravascular clotting (DIC)

Immune overactivation/inflammation:

- Promotes plaque rupture and can contribute to oxygen supply demand mismatch in type 2 myocardial infarction and myocardial injury after noncardiac surgery (MINS)

- Can progress to cytokine storm in some severe cases

Autonomic hyperactivity:

- May induce lethal arrhythmia

- Causes hemodynamic instability:

- Hypertensive crisis

- Vasovagal hypotension and bradycardia

- Responsible for neurogenic stunning that causes or exacerbates heart failure

- Impairs microcirculation, probably both directly and indirectly

High anxiety:

- May worsen the compliance of the patient:

- Weakens the informed consent process

- Impairs periprocedural cooperation of the patient

- Negatively affects the rehabilitation course

Various and unclear mechanisms:

- Causes stress ulcer

- Induces renal failure, plays a role in contrast induced nephropathy (CIN)

Overly prolonged/chronic stress:

- Leads to atherosclerosis, at least through the induction of metabolic syndrome

- Is most likely responsible for impaired wound healing after procedures 
also suggests a clinically relevant relationship between adaptive changes to common stressors such as waking and higher workload, along with circadian fluctuations in allostatic effectors' output [11]. Psychological stress has been shown to induce a prothrombotic state through increases in fibrinogen, von Willebrand factor, and several other serum coagulation factors [2, 11]. Public speaking and even mental arithmetic, such as the serial seven subtraction test, can induce changes in contractility, which predict cardiovascular events [11]. Stressful mental tasks (Stroop task and mirror tracing) have also been shown to induce detectable levels of cardiac troponin T, which were related to the magnitude of the salivary cortisol response to those stressors [33]. Such seemingly mild mental stressors may also cause abnormal perfusion (as assessed by positron emission tomography - PET scanning with rubidium-32) in $75 \%$ of patients with coronary artery disease, similar to one evoked by exercise testing, yet more frequently painless [2]. Moreover, the inflammatory state associated with the stress response contributes to plaque rupture [11, 34]. In addition, intense acute stressors can cause pulmonary embolism, life-threatening tachyarrhythmias and sudden death, as well as inducing stress-related cardiomyopathy syndromes [2, 25].

\section{Psychological aftermath of myocardial infarction}

Psychological distress associated with $\mathrm{MI}$ and its treatment has serious consequences for mental health, which are rarely adequately addressed, counselled and treated. The most important issues are depression and PTSD following MI. The prevalence of depression after $\mathrm{MI}$ is very high, averaging $28.7 \%$ [35]. While personality traits and coping strategies also affect mental health, and type $D$ personality is more often associated with MACE than depression alone [36], antidepressant treatment after $\mathrm{MI}$ reduces the number of hospitalizations and the risk of recurrent $\mathrm{MI}$ [37]. Also, nearly in one in $3(31 \%)$ patients after MI, PTSD is found when a targeted interview is performed [38]. Interventional procedures such as CABG and PCI seem to aggravate the symptoms of PTSD [38], possibly by additional traumatic experience, which may suggest the importance of managing the patient's stress during these procedures. The severity of PTSD is also modulated by personality traits, and especially a neurotic and antagonistic (high in neuroticism and low in agreeableness) personality type exacerbates PTSD symptoms and predisposes to fully symptomatic PTSD development, while maladaptive coping strategies tend to worsen the reported symptoms of other comorbidities [38-40].

\section{Stress induces acute heart failure}

Neurogenic stress cardiomyopathy (NSC) is a well-recognized syndrome complicating the course of acute cerebral events, especially subarachnoid hemorrhage, that can lead to secondary brain damage $[25,41]$. Stress-related cardiomyopathy syndromes that are not associated with organic brain damage have also been identified, and are now commonly called the TTS [3, 25, 42, 43]. In parallel to that, we recently published the first attempt to quantify the incidence of neurogenically stunned myocardium in ACS [4]. We found that one quarter of patients admitted with first-time ACS had regional wall-motion abnormalities (RWMAs) reaching beyond the vasculature of significant coronary stenoses or occurring in a completely unrelated region, or in the absence of any stenosis [4]. These (most likely) neurogenic RWMAs, that occur remotely to the culprit lesion in epicardial arteries, are probably a type of TTS in response to emotional and systemic stress associated with myocardial injury, which has been strikingly poorly recognized thus far. The schematic mechanism of stress-related neurogenic stunning in ACS is shown in Figure 3.

Long-term studies have shown that TTS is not a benign disease, with the all-cause mortality curve markedly worse than matched controls and similar to patients with coronary artery disease - current estimates show a death rate of $5.6 \%$ patient-years and a major adverse cardiovascular event (MACE) rate of $9.9 \%$ patient-years [44]. NSC and TTS have been appropriately linked as having similar clinical presentation, histopathologic findings and most likely also pathophysiology, the only distinction being the presence or absence of structural or infectious brain insult $[25,45]$. The apical-sparing types of these conditions (basal, mid-ventricular and focal) remain largely undetected, due to their less vivid clinical presentation [4]. All these new data, delineating the very elusive and yet apparent brain-heart crosstalk, solidify the exciting emerging field of neurocardiology [4, 25, 46-49].

\section{Stress in endovascular procedures}

The periprocedural stress in elective interventional cardiology is mainly psychological in nature, since the physical insult of endovascular procedures is minimal. However, a patient is also prone to stress associated with allergic reactions and septic complications. Additionally, some procedures may directly elicit the stress reaction through various reflex responses. Conversely to the surgical setting, premedication before smaller procedures is extremely rarely used, while the anxiety level among patients does not seem to be different $[50,51]$. In fact, even peripheral venipuncture for routine diagnostic blood tests is a severe stressor to some individuals [52] that can cause a vasovagal response and unnecessary allostatic load.

On the other hand, recent reports suggest positive results of periprocedural exogenous glucocorticoids also in endovascular procedures such as endovascular aneurysm repair (EVAR) [53]. Furthermore, periprocedural systemic steroids have been found in a few studies to significantly 


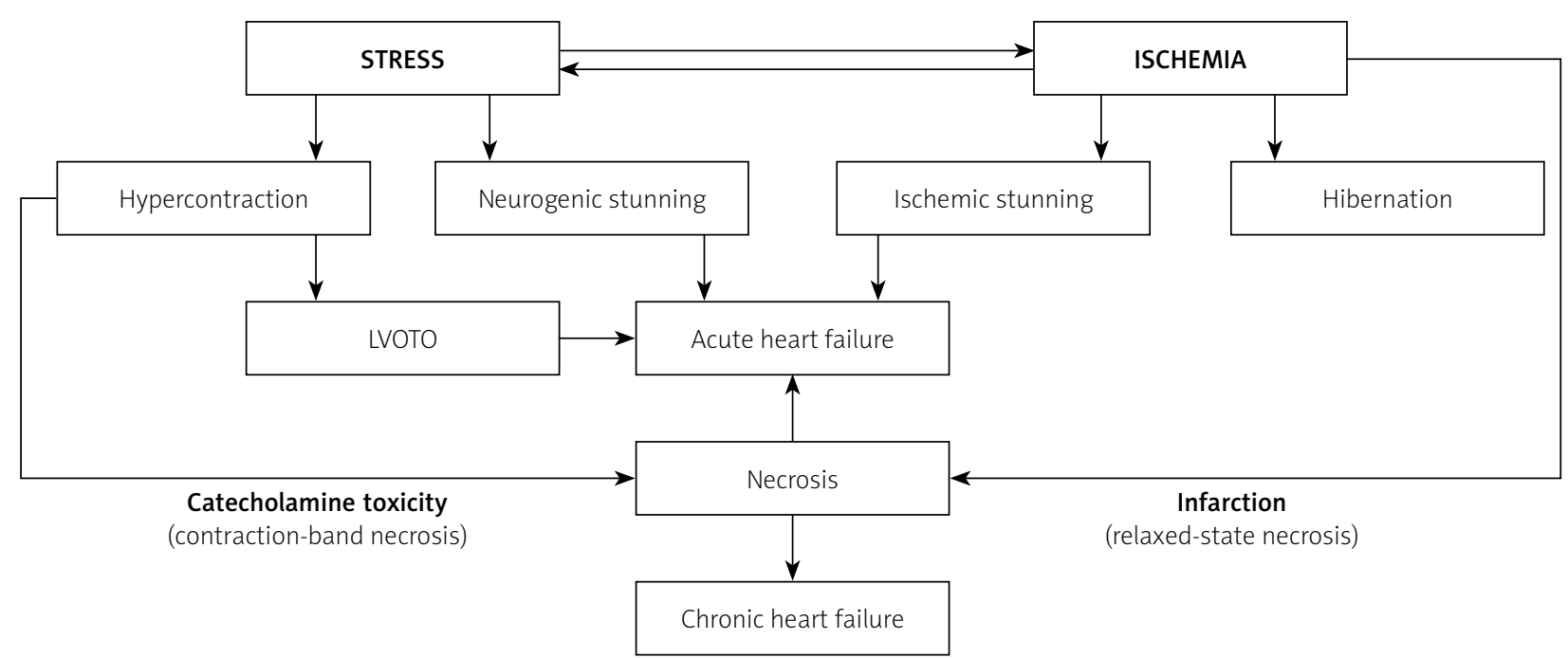

Figure 3. Mechanisms through which stress contributes to contractility impairment in acute coronary syndromes (ACS). Stress-related sympathetic overactivity causes hypercontraction, which results in impaired relaxation, and, if severe, can lead to left ventricular outflow tract obstruction (LVOTO), and to development of contraction-band necrosis (as in takotsubo syndrome). In predisposed individuals (estimated to be one in every 4 patients [4]), sympathetic stimulation can cause neurogenic stunning, and contribute to acute heart failure. Neurogenic stunning can be focal (remote to ischemic insult) or global. Ischemic stunning occurs in the area of impaired perfusion. Hibernation is relatively rare in ACS, but may coexist in chronically and profoundly hypoperfused regions (typically $>85 \%$ stenosis), contributing to chronic ischemic heart failure development and to systolic function recovery, if unfrozen by revascularization. According to Iwaszczuk et al. [4] (modified)

reduce restenosis after coronary bare metal stent (BMS) deployment, with a $28 \%$ reduction in all-cause mortality at follow-up, albeit statistically insignificant [54]. Another type of stress associated with endovascular procedures is the reflex hypotension commonly encountered in carotid artery stenting [55].

\section{Surgical stress}

The stress response induces insulin resistance and a catabolic state following a surgical insult, leading to, inter alia, stress hyperglycemia [56]. This catabolic state, aggravated by the routine of preoperative fasting, is proven to be detrimental for surgical outcomes, and preoperative oral carbohydrate loading shows promising results, especially in major surgery [56]. Physical trauma is likely to produce changes in immunity through vast connections of somatic sensory nerves and the immunological system [26]; however, if not prolonged, it is believed to be beneficial rather than detrimental overall, enhancing hemostasis and reducing the risk of immunization to own antigens that are hidden from the immune system in the absence of trauma $[1,6]$. The excess preoperative anxiety is shown to be detrimental [57] and probably associated with HPA axis overactivity, as in most instances of psychological stress $[10,15]$. Higher HPA axis activity independently predicts the occurrence of delirium after cardiac surgery $[58,59]$. Surgery-related PTSD occurs especially, but not exclusively, in patients with intraoperative awareness, but HPA axis overactivity has also been associated with this phenomenon [30,60]. There is also substantial evidence linking psychological stress and associated higher cortisol levels to impaired wound healing $[6,61]$. We also found an association of preoperative anxiety before a vascular surgery procedure and the occurrence of infectious complications within 30 days after surgery [50]. On the other hand, perioperative glucocorticoid administration has been shown to provide benefit and better recovery from cardiac, liver, and orthopedic (and other) procedures without any significant complications, including the risk of infection [62-65]. Conversely, the routine use of "stress dose" glucocorticoids in the perioperative period in patients with chronic glucocorticoid immunosuppression (and therefore potential adrenal insufficiency due to atrophy of the glands) has recently been found to be obsolete [66-68]. The possible explanation of these, seemingly conflicting, results is that a strong physiologic perioperative stress response (as measured by endogenous cortisol levels) is mainly detrimental, but a short course of exogenous glucocorticoids has the potential to alleviate this reaction (since cortisol is the main player in the feedback loop initiating reflex termination of the stress response $[1,15,50])$, whereas they are mostly unnecessary in patients whose adrenals are suppressed. Therefore, perioperative exogenous glucocorticoids may not necessarily mimic and potentiate the endogenous stress response, but rather serve as a means 
of controlling it. This hypothesis, however, needs further research to confirm it.

Surgical stress is further accompanied by the anesthesia-related stress caused by anesthesia induction, tracheal reflex from intubation or drugs used intraoperatively, that can (among other things) disrupt the immune system through mastocyte activation, all of which may lead to refractory stress cardiomyopathy and SCD [25].

\section{Periprocedural myocardial injury: MI and MINS}

Over 200 million surgical procedures are performed worldwide each year and the prevalence of perioperative myocardial insult of any cause is estimated at $18 \%$ (previously found in $8 \%$ using $4^{\text {th }}$ generation troponin $T$ measurements) and has been called myocardial injury after noncardiac surgery (MINS) [69-71]. Only ca. $21 \%$ of these patients met the diagnostic criteria of the $3^{\text {rd }}$ Universal Definition of $\mathrm{Ml}$; the others are considered to suffer from less severe ischemic cardiac injury [71]. There are, however, reasons to believe that a large proportion of these patients reflect non-ischemic cardiac injury in the form of perioperative stress cardiomyopathy, yet directed studies are needed to confirm this hypothesis. Perioperative disturbances in the hemostatic system may also play a role [72]. Resolving this issue is indeed of great importance, since MINS is associated with increased postoperative mortality [69-71, 73], and accurate diagnosis is essential for proper treatment. Currently, MINS is defined as an acute increase in the concentration of cardiac troponin during or within 30 days after non-cardiac surgery, in the absence of a documented non-coronary primary cause of troponin elevation (e.g., sepsis, pulmonary embolism) [73]. For $5^{\text {th }}$ generation high-sensitivity troponin $T$ measurements, present criteria require a postoperative level of $\geq 65 \mathrm{ng} / \mathrm{l}$ or in the range of 20-64 $\mathrm{ng} / \mathrm{l}$ with an absolute postoperative change of at least $5 \mathrm{ng} / \mathrm{ml}[70$, $71,73]$. We recently reported that MINS is more common, and therefore a greater issue, than DVT/PE after vascular surgery [70]. To date, there are no reliable data regarding the equivalent of MINS in interventional cardiology, but the current $4^{\text {th }}$ Universal Definition of $\mathrm{MI}$ recommends recognition of myocardial injury whenever troponin levels exceed the normal range (i.e., the $99^{\text {th }}$ percentile of the healthy population, which corresponds to > $14 \mathrm{ng} / \mathrm{l}$ of troponin T) [74].

\section{Therapeutic interventions}

Minimization of stressful stimuli that accompany the procedure is very important and often neglected in hospital routines [57]. Many such interventions are obvious and self-explanatory, such as not exposing the patient to unnecessary social stress related to one's nudity or providing thermal comfort, and therefore become neglected in formal regulations. We are not arguing that they should otherwise, but still, there must be adequate re- sources to fulfill those simple tasks, such as appropriate gowns or blankets need to be readily available when and where they are required.

\section{Abandon fasting}

Preprocedural fasting promotes a catabolic state and insulin resistance, which further exacerbates catabolic reactions associated with tissue injury, especially after major surgery [56], and hunger is a potent stressor. Oral carbohydrate loading has been shown to promote faster recovery after major surgery $[56,75]$, while fasting seems generally obsolete prior to coronary angiography and other endovascular procedures [75].

\section{Stress-reducing medication}

Pain control is an issue of utmost importance, but the vast literature on adequate pain control lies beyond the scope of this review and can be found summarized elsewhere [76].

Hypnotics such as benzodiazepines act quickly and effectively; however, serious side effects limit their routine use in sufficient doses, especially when patient cooperation is needed. Second generation antipsychotics (especially quetiapine) seem particularly useful in older patients with dementia, and are relatively safe in the short-term use. Antihistamines are preferable in younger patients due to their relative efficacy and good safety profile (maximal daily dose of hydroxyzine equals $400 \mathrm{mg}$, and the recommended initial dosage in adult periprocedural premedication is $100 \mathrm{mg}$ ). The patient after hydroxyzine administration usually remains cooperative, while its antihistaminic action has the potential to alleviate possible allergic responses, nausea and vomiting that may occur during the procedure. Antidepressants, due to their delayed onset of action, are preferred for follow-up treatment in selected individuals with high anxiety. Selective serotonin reuptake inhibitors (SSRI) appear as first-line treatment, and especially sertraline because of its efficacy and lowest risk of cardiac complications. Bupropion, although not as safe as SSRIs, may be considered in highly selected patients due to its powerful potential to facilitate smoking cessation and weight loss. Recent pooled data show a robust reduction in rehospitalization rates and in the risk of recurrent MI with antidepressants administration after ACS, which further sanctions their use [37]. Other useful medications include pregabalin, buspirone and certain herbal medications such as lavender oil [77]. $\beta$-blockers, especially lipophilic formulations, also exert anxiolytic effects, although they may need to be paired with antidepressants for longterm interventions, because of their long-recognized potential to induce depression.

Interestingly, newest data suggest that angiotensin-converting enzyme inhibitors (ACEI) are the only class of drugs providing protection from TTS recurrence [44]. This is of note, since angiotensin II is, apart from 
being a vasoconstrictor, also a neurotransmitter that is projected from brain chemoreceptors to the dorsomedial hypothalamus, initiating a stress reaction, especially in response to systemic stressors (Figure 2).

\section{Psychological interventions}

Psychological interventions in coronary heart disease were found to reduce cardiovascular mortality as well as measures of psychological well-being, but without affecting total mortality or recurrence of non-fatal MI [78]. Preoperative psychological preparation before surgery seems to lower the postoperative pain, shorten recovery and hospital stay, as well as to reduce negative affect, but the quality of evidence is judged as low, due to high probability of bias [79]. In children undergoing cardiac surgery, significantly lower anxiety, pain and cortisol levels, as well as better mood, were noted when they were provided with toys and video games prior to the procedure, while their parents benefited from more detailed information and counselling [80]. Maternal anxiety tends to be higher than paternal anxiety, and is linked with estimated periprocedural risk, as well as codependent on the spouse's anxiety level [81], probably reflecting the level of emotional support in the family. Adults undergoing elective percutaneous coronary intervention ( $\mathrm{PCl}$ ) benefited from mindfulness-based psychological interventions, which improved quality of life and reduced anxiety, stress and depressiveness in patients under the age of 60 [82]; moreover, such interventions were proven effective even in the form of brief one-on-one telephone calls [83]. Also, stress management training added to cardiac rehabilitation programs is associated with significantly better functioning and fewer cardiovascular events compared to exercise-only-based rehabilitation [84].

\section{Conclusion - friend or foe?}

Patient stress responses in the periprocedural period may be both beneficial and detrimental. The majority of scientific reports appear to focus on the harmful effects of stress. In contrast, it is not routinely understood (or appreciated) to what extent periprocedural bleeding or other complications are prevented owing to the patient's intrinsic stress regulatory mechanisms. This is because we have insufficient means of measuring, understanding, and controlling even the major pathways contributing to the allostatic network, not even during general anesthesia. At this stage, we must assume that the constellation of those most powerful regulatory mechanisms in the body, preserved and built upon by natural selection throughout almost all phylogenetic lines in the animal kingdom, plays the most important role in deciding whether the patient is going to survive the acute phase of any disease. Expanding the knowledge about mechanisms of the stress response has great potential to eventually unravel and harness these powerful regu- latory systems, and to modulate their action to achieve therapeutic goals. All in all, periprocedural patient stress is a difficult friend, which offers important mechanisms enhancing survival and recovery, but, due to its many dark sides, may also turn into a foe.

\section{Further research}

Further research is needed to disentangle, understand and learn to modulate the allostatic network of the stress response as a tool to optimize procedural outcomes. Major areas for further research include: 1) determination of detrimental and positive aspects of various shades of stress responses in different medical settings; 2) development of clinically useful tools to easily, quickly and reliably determine "bad" aspects of stress in a patient; 3) randomized controlled trials assessing the effects of adequately timed and targeted intervention on specific aspects of stress effectors' under- or overactivation, which has been proven detrimental, rather than aiming to tackle the issue too broadly, such as "lowering" or "enhancing the stress response" in general.

\section{Acknowledgments}

The corresponding author received support for this work from the Polish Ministry of Science and Higher Education, grant no. DI 2011024141.

\section{Conflict of interest}

The authors declare no conflict of interest.

\section{References}

1. McEwen BS. Physiology and neurobiology of stress and adaptation: central role of the brain. Physiol Rev 2007; 87: 873-904.

2. Dimsdale JE. Psychological stress and cardiovascular disease. J Am Coll Cardiol 2008; 51: 1237-46.

3. Yoshikawa T. Takotsubo cardiomyopathy, a new concept of cardiomyopathy: clinical features and pathophysiology. Int J Cardiol 2015; 182: 297-303.

4. Iwaszczuk P, Kołodziejczyk B, Kruczek T, et al. Ischemic versus non-ischemic (neurogenic) myocardial contractility impairment in acute coronary syndromes: prevalence and impact on left ventricular systolic function recovery. Med Sci Monit 2018; 24: 3693-701.

5. Neeman E, Ben-Eliyahu S. Surgery and stress promote cancer metastasis: new outlooks on perioperative mediating mechanisms and immune involvement. Brain Behav Immun 2013; 30: S32-40.

6. Dhabhar FS. Effects of stress on immune function: the good, the bad, and the beautiful. Immunol Res 2014; 58: 193-210.

7. Sood P, Priyadarshini S, Aich P. Psychological stressors as interventions: good out of the evil. Front Biosci 2012; 4: 43-60.

8. Siervo M, Wells JCK, Cizza G. The contribution of psychosocial stress to the obesity epidemic: an evolutionary approach. Horm Metab Res 2009; 41: 261-70.

9. Moreira MCS, Pinto ISJ, Mourão AA et al. Does the sympathetic nervous system contribute to the pathophysiology of metabolic syndrome? Front Physiol 2015; 6: 234 
10. Łosiak W. Psychologia stresu. Wydawnictwa Akademickie i Profesjonalne, Warszawa 2008.

11. Schwartz BG, French WJ, Mayeda GS, et al. Emotional stressors trigger cardiovascular events. Int J Clin Pract 2012; 66: 631-9.

12. Keel M, Trentz O. Pathophysiology of polytrauma. Injury 2005; 36: 691-709.

13. Harper D. stress, distress, strain. Online Etymology Dict. http:// www.etymonline.com/index.php (accessed October 11, 2015).

14. Tofield A. Connecting the pieces of Takotsubo "broken heart". Eur Heart J 2018; 39: 2021-2.

15. Ulrich-Lai YM, Herman JP. Neural regulation of endocrine and autonomic stress responses. Nat Rev Neurosci 2009; 10: 397-409.

16. Gonzalez A, Zvolensky MJ, Hogan J, et al. Anxiety sensitivity and pain-related anxiety in the prediction of fear responding to bodily sensations: a laboratory test. J Psychosom Res 2011; 70 : 258-66.

17. McEwen BS, Wingfield JC. The concept of allostasis in biology and biomedicine. Horm Behav 2003; 43: 2-15

18. Ikeoka D, Mader JK, Pieber TR. Adipose tissue, inflammation and cardiovascular disease. Rev Assoc Med Bras 2010; 56: 116-21.

19. Bruce MA, Griffith DM, Thorpe RJ. Stress and the kidney. Adv Chronic Kidney Dis 2015; 22: 46-53.

20. Bellavance MA, Rivest S. The HPA - immune axis and the immunomodulatory actions of glucocorticoids in the brain. Front Immunol 2014; 5: 136.

21. Sapolsky RM, Romero LM, Munck AU. How do glucocorticoids influence stress responses? Integrating permissive, suppressive, stimulatory, and preparative actions. Endocr Rev 2000; 21: 55-89.

22. Rang HP, Dale MM, Ritter JM, et al. Rang and Dale's Pharmacology. $7^{\text {th }}$ ed. Elsevier Inc Curchill Livingstone; Edinburgh 2012.

23. Hirsch D, Zukowska Z. NPY and stress 30 years later: the peripheral view. Cell Mol Neurobiol 2012; 32: 645-59.

24. Burnstock G. Purinergic signalling: pathophysiology and therapeutic potential. Keio J Med 2013; 62: 63-73.

25. Mazzeo AT, Micalizzi A, Mascia L, et al. Brain-heart crosstalk: the many faces of stress-related cardiomyopathy syndromes in anaesthesia and intensive care. Br J Anaesth 2014; 112: 803-15.

26. Ordovas-Montanes J, Rakoff-Nahoum S, Huang S, et al. The regulation of immunological processes by peripheral neurons in homeostasis and disease. Trends Immunol 2015; 36: 578-604.

27. Arora S, Sevdalis N, Nestel D, et al. The impact of stress on surgical performance: a systematic review of the literature. Surgery 2010; 147: 318-30.e6.

28. Georgiou K, Larentzakis A, Papavassiliou AG. Surgeons' and surgical trainees' acute stress in real operations or simulation: a systematic review. Surg 2017; 15: 355-65.

29. Allolio B. Adrenal crisis. Eur J Endocrinol 2015; 172: R115-24.

30. Porhomayon J, Kolesnikov S, Nader ND. The impact of stress hormones on post-traumatic stress disorders symptoms and memory in cardiac surgery patients. J Cardiovasc Thorac Res 2014; 6: 79-84.

31. Rozanski A, Blumenthal JA, Davidson KW, et al. The epidemiology, pathophysiology, and management of psychosocial risk factors in cardiac practice: the emerging field of behavioral cardiology. J Am Coll Cardiol 2005; 45: 637-51.

32. Wittstein IS, Thiemann DR, Lima JAC, et al. Neurohumoral features of myocardial stunning due to sudden emotional stress. N Engl J Med 2005; 352: 539-48.

33. Lazzarino Al, Hamer M, Gaze D, et al. The association between cortisol response to mental stress and high-sensitivity cardiac troponin T plasma concentration in healthy adults. J Am Coll Cardiol 2013; 62: 1694-701.

34. Devereaux PJ, Goldman L, Cook DJ, et al. Perioperative cardiac events in patients undergoing noncardiac surgery: a review of the magnitude of the problem, the pathophysiology of the events and methods to estimate and communicate risk. CMAJ 2005; 173: 627-34.

35. Feng L, Li L, Liu W, et al. Prevalence of depression in myocardial infarction. Medicine 2019; 98: e14596.

36. Du J, Zhang D, Yin Y, et al. The personality and psychological stress predict major adverse cardiovascular events in patients with coronary heart disease after percutaneous coronary intervention for five years. Medicine 2016; 95: e3364.

37. Sweda R, Siontis GCM, Nikolakopoulou A, et al. Antidepressant treatment in patients following acute coronary syndromes: a systematic review and Bayesian meta-analysis. ESC Hear Fail 2020; 7: 3610-20.

38. Chung MC, Berger Z, Rudd H. Coping with posttraumatic stress disorder and comorbidity after myocardial infarction. Compr Psychiatry 2008; 49: 55-64.

39. Chung MC, Dennis I, Berger Z, et al. Posttraumatic stress disorder following myocardial infarction: personality, coping, and trauma exposure characteristics. Int J Psychiatry Med 2011; 42: 393-419.

40. Chung MC, Berger Z, Rudd H. Comorbidity and personality traits in patients with different levels of posttraumatic stress disorder following myocardial infarction. Psychiatry Res 2007; 152: 243-52.

41. Wybraniec MT, Mizia-Stec K, Krzych $Ł$. Neurocardiogenic injury in subarachnoid hemorrhage: a wide spectrum of catecholamin-mediated brain-heart interactions. Cardiol J 2014; 21: 220-8.

42. Redfors B, Shao Y, Lyon AR, et al. Diagnostic criteria for takotsubo syndrome: a call for consensus. Int J Cardiol 2014; 176: 274-6.

43. Ghadri JR, Wittstein IS, Prasad A, et al. International Expert Consensus Document on takotsubo syndrome (part i): clinical characteristics, diagnostic criteria, and pathophysiology. Eur Heart J 2018; 39: 2032-46.

44. Ghadri JR, Wittstein IS, Prasad A, et al. International Expert Consensus Document on takotsubo syndrome (part ii): diagnostic workup, outcome, and management. Eur Heart J 2018; 39: 2047-62.

45. Wybraniec M, Mizia-Stec K, Krzych L. Stress cardiomyopathy: yet another type of neurocardiogenic injury: "stress cardiomyopathy”. Cardiovasc Pathol 2014; 23: 113-20.

46. Templin C, Hänggi J, Klein C, et al. Altered limbic and autonomic processing supports brain-heart axis in Takotsubo syndrome. Eur Heart J 2019; 40: 1183-7.

47. Ghadri JR, Levinson RA, Lüscher TF, et al. Neurocardiology: the brain-heart connection in Takotsubo syndrome. Eur Heart J 2019; 40: 3062-3.

48. Chen Z, Venkat P, Seyfried D, et al. Brain-heart interaction. Circ Res 2017; 121: 451-68.

49. Zou R, Shi W, Tao J, et al. Neurocardiology: cardiovascular changes and specific brain region infarcts. Biomed Res Int 2017; 2017 : 5646348.

50. Iwaszczuk P. The role of preoperative stress, measured with anxiety questionnaires (STAI, APAIS) and physiological markers (CORTISOL, IL-6), on vascular surgery course and complications. Jagiellonian University 2013. 
51. Roohafza H, Sadeghi $M$, Khani A, et al. Psychological state in patients undergoing coronary artery bypass grafting surgery or percutaneous coronary intervention and their spouses. Int J Nurs Pract 2015; 21: 214-20.

52. Gonzalez A, Zvolensky MJ, Hogan J, et al. Anxiety sensitivity and pain-related anxiety in the prediction of fear responding to bodily sensations: a laboratory test. J Psychosom Res 2011; 70: 258-66.

53. de la Motte L, Schroeder TV, Kehlet H. Should steroids be used during endovascular aortic repair? Adv Surg 2015; 49: 173-84.

54. Sardar P, Chatterjee S, Mukherjee D, et al. Steroids for the prevention of restenosis in bare-metal stents: a systematic review and meta-analysis. J Invasive Cardiol 2012; 24: 98-103.

55. Mazurek A, Borratynska A, Malinowski KP, et al. MicroNET-covered stents for embolic prevention in patients undergoing carotid revascularisation: twelve-month outcomes from the PARADIGM study. Eurolntervention 2020; 16: e950-2.

56. Bilku D, Dennison A, Hall T, et al. Role of preoperative carbohydrate loading: a systematic review. Ann R Coll Surg Engl 2014; 96: 15-22.

57. Ayyadhah Alanazi A. Reducing anxiety in preoperative patients: a systematic review. Br J Nurs 2014; 23: 387-93.

58. Kazmierski J, Banys A, Latek J, et al. Cortisol levels and neuropsychiatric diagnosis as markers of postoperative delirium: a prospective cohort study. Crit Care 2013; 17: R38.

59. Cerejeira J, Batista P, Nogueira V, et al. The stress response to surgery and postoperative delirium: evidence of hypothalamic-pituitary-adrenal axis hyperresponsiveness and decreased suppression of the GH/IGF-1 axis. J Geriatr Psychiatry Neurol 2013; 26: 185-94.

60. Whitlock EL, Rodebaugh TL, Hassett AL, et al. Psychological sequelae of surgery in a prospective cohort of patients from three intraoperative awareness prevention trials. Anesth Analg 2015; 120: 87-95.

61. Walburn J, Vedhara K, Hankins M, et al. Psychological stress and wound healing in humans: a systematic review and meta-analysis. J Psychosom Res 2009; 67: 253-71.

62. Liu C, Wang J, Yiu D, et al. The efficacy of glucocorticoids for the prevention of atrial fibrillation, or length of intensive care unite or hospital stay after cardiac surgery: a meta-analysis. Cardiovasc Ther 2014; 32: 89-96.

63. Orci LA, Toso C, Mentha G, et al. Systematic review and metaanalysis of the effect of perioperative steroids on ischaemia-reperfusion injury and surgical stress response in patients undergoing liver resection. Br J Surg 2013; 100: 600-9.

64. Yue C, Wei R, Liu Y. Perioperative systemic steroid for rapid recovery in total knee and hip arthroplasty: a systematic review and meta-analysis of randomized trials. J Orthop Surg Res 2017; 12: 100 .

65. Toner AJ, Ganeshanathan V, Chan MT, et al. Safety of perioperative glucocorticoids in elective noncardiac surgery: a systematic review and meta-analysis. Anesthesiology 2017; 126: 234-48.

66. Kelly KN, Domajnko B. Perioperative stress-dose steroids. Clin Colon Rectal Surg 2013; 26: 163-7.

67. MacKenzie CR, Goodman SM. Stress dose steroids: myths and perioperative medicine. Curr Rheumatol Rep 2016; 18: 47.

68. Hamrahian AH, Fleseriu M, AACE Adrenal Scientific Committee. Evaluation and management of adrenal insufficiency in critically ill patients: disease state review. Endocr Pract 2017; 23: 716-25.

69. Botto F, Alonso-Coello P, Chan MTV, et al. Myocardial injury after noncardiac surgery: a large, international, prospective cohort study establishing diagnostic criteria, characteristics, predictors, and 30-day outcomes. Anesthesiology 2014; 120: 564-78.

70. Górka J, Polok K, Fronczek J, et al. Myocardial injury is more common than deep venous thrombosis after vascular surgery and is associated with a high one year mortality risk. Eur J Vasc Endovasc Surg 2018; 56: 264-70.

71. Writing Committee for the VISION Study Investigators, Devereaux PJ, Biccard BM, Sigamani A, et al. Association of postoperative high-sensitivity troponin levels with myocardial injury and 30-day mortality among patients undergoing noncardiac surgery. JAMA 2017; 317: 1642-51.

72. Polok KJ, Górka J, Fronczek J, et al. Impact of arterial procedures on coagulation and fibrinolysis - a pilot study. Brazil J Cardiovasc Surg 2019; 34: 327-34.

73. Devereaux PJ, Szczeklik W. Myocardial injury after non-cardiac surgery: diagnosis and management. Eur Heart J 2020; 41: 3083-91.

74. Thygesen K, Alpert JS, Jaffe AS, et al. Fourth Universal Definition of Myocardial Infarction (2018). Glob Heart 2018; 13: 305-38.

75. Aguilar-Nascimento JE de, Feguri GR. Fasting may not be required before percutaneous coronary intervention. Evid Based Nurs 2015; 18: 41.

76. Mitra S, Carlyle D, Kodumudi G, et al. New advances in acute postoperative pain management. Curr Pain Headache Rep 2018; 22: 35 .

77. Strawn JR, Geracioti L, Rajdev N, et al. Pharmacotherapy for generalized anxiety disorder in adult and pediatric patients: an evidence-based treatment review. Expert Opin Pharmacother 2018; 19: 1057-70.

78. Richards SH, Anderson L, Jenkinson CE, et al. Psychological interventions for coronary heart disease. Cochrane Database Syst Rev 2017; 4: CD002902.

79. Powell R, Scott NW, Manyande A, et al. Psychological preparation and postoperative outcomes for adults undergoing surgery under general anaesthesia. Cochrane Database Syst Rev 2016; 5: CD008646.

80. Kumar A, Das S, Chauhan S, et al. Perioperative anxiety and stress in children undergoing congenital cardiac surgery and their parents: effect of brief intervention - a randomized control trial. J Cardiothorac Vasc Anesth 2019; 33: 1244-50.

81. Werner O, El Louali F, Fouilloux V, et al. Parental anxiety before invasive cardiac procedure in children with congenital heart disease: contributing factors and consequences. Congenit Heart Dis 2019; 14: 778-84.

82. Nykliček I, Dijksman SC, Lenders PJ, et al. A brief mindfulness based intervention for increase in emotional well-being and quality of life in percutaneous coronary intervention $(\mathrm{PCl})$ patients: the MindfulHeart randomized controlled trial. J Behav Med 2014; 37: 135-44.

83. Hou Y, Zhao X, Lu M, et al. Brief, one-on-one, telephone-adapted mindfulness-based stress reduction for patients undergoing percutaneous coronary intervention: a randomized controlled trial. Transl Behav Med 2019; 9: 1216-23.

84. Lavie CJ, Menezes AR, De Schutter A, et al. Impact of cardiac rehabilitation and exercise training on psychological risk factors and subsequent prognosis in patients with cardiovascular disease. Can J Cardiol 2016; 32: S365-73. 